University of Windsor

Scholarship at UWindsor

1971

\title{
Theory of relativistic magnetic dipole transitions: Lifetime of the metastable 2S3 state of the heliumlike ions
}

Gordon W. F. Drake

University of Windsor

Follow this and additional works at: https://scholar.uwindsor.ca/physicspub

Part of the Physics Commons

\section{Recommended Citation}

Drake, Gordon W. F.. (1971). Theory of relativistic magnetic dipole transitions: Lifetime of the metastable 2S3 state of the heliumlike ions. Physical Review A, 3 (3), 908-915.

https://scholar.uwindsor.ca/physicspub/114

This Article is brought to you for free and open access by the Department of Physics at Scholarship at UWindsor. It has been accepted for inclusion in Physics Publications by an authorized administrator of Scholarship at UWindsor. For more information, please contact scholarship@uwindsor.ca. 
not possible to note any tendency towards an increase or a decrease of this ratio with the atomic number.

A decrease in the slope of the $K_{\beta} / K_{\alpha}-v s-Z$ curve was observed by Hansen et al. ${ }^{2}$ in the lanthanide region. Tentatively this was attributed to the influence of filling the $4 f$ shell. The same effect would be expected in the actinide region where the $5 f$ shell is being filled. In this region, however, we have only two points, for Th and $\mathrm{U}$, and here the $K_{\beta} / K_{\alpha}$ ratios are equal within experimental errors. In fact, this ratio was carefully measured in mercury, giving $0.275 \pm 0.004$. It rises gradually up to $0.284 \pm 0.005$ in thorium and $0.283 \pm 0.005$ in uranium. This feature of the $x$-ray spectrum is very interesting and should be studied in greater detail.

In these measurements the most important source of experimental error is due to the efficiency curve of the detector. The spread in energy between the $K L_{I I}$ and the $K O_{I I I} \mathrm{x}$ rays is about $14 \mathrm{keV}$ in mercury and $21 \mathrm{keV}$ in uranium. It is hard to reduce the experimental error due to efficiency differences below $1 \%$ for the first interval and $1.5 \%$ for the second.

An attempt was made to resolve the transitions from the $M_{\mathrm{IV}}-M_{\mathrm{V}}$ and $N_{\mathrm{III}}-N_{\mathrm{IV}-\mathrm{V}}$ subshells in the heavier elements. In $\mathrm{Ra}$ and $\mathrm{U}$ we found $K M_{\mathrm{IV}} / K M_{\mathrm{V}}$ $=1.1 \pm 0.3$ and $K N_{I V-V} / K N_{I I I}=0.08 \pm 0.03$. The first value is in good agreement with the theory.

Note added in proof. In a recently published work Hansen et al. [Nucl. Phys. A153, 465 (1970)] measured relative $K \mathrm{x}$-ray transition probabilities at $Z=96$ from ${ }^{249} \mathrm{Cf}$ decay. The reported values $K L_{\text {II }} / K L_{\text {III }}=0.626 \pm 0.006$ and $K_{\beta} / K_{\alpha}=0.327 \pm 0.010$ are slightly below and above the calculated ${ }^{1}$ values, respectively. This agrees with the general tendency shown by our measurements.

\section{ACKNOWLEDGMENT}

We should like to express our thanks to E. F. da Silveira for his help in the early stages of this work.
*Work supported by grants from Conselho Nacional de Pesquisas and Banco Nacional de Desenuolvimento Economico (FUNTEC-23).

1J. H. Scofield, Phys. Rev, 179,9 (1969).

${ }^{2} J$. S. Hansen, H. V. Freund, and R. W. Fink, Nucl. Phys. A142, 604 (1970).
${ }^{3}$ A. G. de Pinho, E. F. da Silveira, and N. L. da Costa, Phys. Rev. C 2,572 (1970).

${ }^{4}$ S. I. Salem, B. G. Saunders, and G. C. Nelson, Phys. Rev. A 1, 1563 (1970).

${ }^{5}$ O. Beckman, Arkiv. Fysik $\underline{9}, 495$ (1955).

\title{
Theory of Relativistic Magnetic Dipole Transitions: Lifetime of the Metastable $2{ }^{3} S$ State of the Heliumlike Ions
}

\author{
G. W. F. Drake \\ Department of Physics, University of Windsor, Windsor, Ontario, Canada \\ (Received 16 September 1970)
}

\begin{abstract}
It has recently been established that the radiative lifetime of the metastable $2^{3} S$ state of helium and the heliumlike ions is determined by single-photon magnetic dipole (M1) transitions to the ground state, rather than the two-photon process proposed by Breit and Teller. The theory of $n l-n^{\prime} l M 1$ transitions with $n \neq n^{\prime}$ is developed in the Pauli approximation and extended to two-electron systems. Terms arising from relativistic energy corrections and finite-wavelength effects are included. The results for hydrogenic systems are shown to be identical to those obtained in the relativistic four-component Dirac formulation. The coefficients in the $Z^{-1}$ perturbation expansion of the $1 s 2 s^{3} s-1 s^{21} S M 1$ transition integral are evaluated through ninth order and used to calculate the $M 1$ emission probabilities from the $2{ }^{3} \mathrm{~S}$ state of the two-electron ions up to $\mathrm{Fe} \mathrm{xxv}$. The emission probability for neutral helium is $1.27 \times 10^{-4} \mathrm{sec}^{-1}$. The results are compared with recent solar coronal observations by Gabriel and Jordan, and with a measurement of the $2{ }^{3} S$ state lifetime in Ar xvII by Schmieder and Marrus.
\end{abstract}

\section{INTRODUCTION}

The radiative lifetime of the metastable $1 s 2{ }^{3} \mathrm{~S}$ state of helium and the heliumlike ions has been a subject of controversy for some time, despite its importance in a variety of astrophysical problems. ${ }^{1}$ Breit and Teller ${ }^{2}$ suggested that the state decays primarily by two-photon electric dipole (2E1) emission, incorrectly estimating the single-photon magnetic dipole $(M 1)$ process to be much slower. A calculation by Mathis ${ }^{3}$ yielded $2.2 \times 10^{-5} \mathrm{sec}^{-1}$ for the $2 E 1$ decay rate, a value used in the astrophysical literature for many years. However, Drake and Dalgarno ${ }^{4}$ showed Mathis's calculation to be 
based on an incorrect formulation of the problem, and detailed calculations by Drake, Victor, and Dalgarno $0^{5}$ and by Bely and Faucher ${ }^{6}$ reduced the $2 E 1$ decay rate to $4 \times 10^{-9} \mathrm{sec}^{-1}$.

Gabriel and Jordan ${ }^{1,7}$ stimulated further theoretical work when they identified lines in the spectrum of the solar corona as the $1 s 2 s^{3} S-1 s^{2}{ }^{1} S$ transition of the heliumlike ions $\mathrm{Cv}$ to Si xIII. The above authors proposed that, contrary to Breit and Teller's conclusion, the $2^{3} S$ state decays primarily by singlephoton magnetic dipole emission. Griem ${ }^{8}$ then demonstrated that results roughly consistent with the coronal observations could be obtained in the Dirac relativistic formulation ${ }^{9}$ with wave functions approximated by hydrogenic products of Dirac spinors.

In this paper, we evaluate certain "finite-wavelength" contributions to the leading term of the $M 1$ transition integral expanded in powers of the finestructure constant $\alpha$, which have been omitted in previous calculations. ${ }^{2,8}$ Further, it is shown that an accurate calculation of the $M 1$ decay rate using correlated nonrelativistic wave functions is possible if the problem is transformed into the Pauli approximation. The contribution to the transition integral from the leading term in $\alpha$ vanishes and it is necessary to retain terms of higher order than those appearing in conventional radiation theory. The higher-order corrections are derived and the general Pauli theory of $n l-n^{\prime} l M 1$ transitions with $n \neq n^{\prime}$ is developed for one- and two-electron systems. (Transitions with $n=n^{\prime}$ do not require higherorder terms to obtain a nonzero result.) A $Z^{-1}$ expansion technique described previously ${ }^{10,11}$ is used to calculate to high order the perturbation expansion coefficients in the $1 s 2 s^{3} S-1 s^{2}{ }^{1} S$ transition integral. $M 1$ decay rates are presented for all the heliumlike ions through Fe xxv.

In the absence of nuclear spin, the $M 1$ process is the only possible single-photon decay mode from the $2{ }^{3} S$ state, since radiation to the ground ${ }^{1} S$ state involves a $J=1 \rightarrow 0$ transition with no parity change. The most efficient processes induced by a finite nuclear spin are the following: electric quadrupole (E2) decay due to the mixing of ${ }^{1} D_{2}^{e}$ states with the $2{ }^{3} S_{1}$ state; and $M 1$ decay due to the intermixing of the $2{ }^{3} S_{1}$ and $1{ }^{1} S_{0}$ states. As shown in the appendix, these processes are slower by factors of $O\left(m^{2} /(Z M)^{2}\right)$ and may be neglected.

The first direct measurement of the lifetime of a $2^{3} \mathrm{~S}$ state has recently been reported in heliumlike Ar xvir by Schmieder and Marrus, ${ }^{12}$ using the Berkeley HILAC as an ion source in a beam-foil experiment. Their measured mean life $172 \pm 30$ nsec provides an important test of the theory.

\section{GENERAL FORMULATION}

The calculation of magnetic dipole transitions is formulated in terms of the theory of the spherical photon as described by Akhiezer and Berestetskii. ${ }^{9}$ Applications to magnetic dipole transitions with $n=n^{\prime}$ are further discussed by Mizushima. ${ }^{13}$ Briefly, the vector potential for a photon in a state of magnetic type with angular momentum $L$, component $M$, and parity $(-1)^{L+1}$ is

$$
\overrightarrow{\mathrm{A}}_{\omega L M}=\vec{a}_{\omega L M}+\vec{a}^{*}{ }_{\omega L M},
$$

where

$$
\begin{aligned}
\overrightarrow{\mathrm{a}}_{\omega L M} & =(\hbar \omega / 4 \pi R)^{1 / 2} \overrightarrow{\mathrm{a}}_{L M} e^{-i \omega t}, \\
\overrightarrow{\mathrm{a}}_{L M} & =g_{L}(\omega r / c) \overrightarrow{\mathrm{Y}}_{L L M}(r)
\end{aligned}
$$

in units with $4 \pi \epsilon_{0}=1 . R$ is the radius of a spherical normalizing volume, $\hbar \omega$ is the photon energy, $\overrightarrow{\mathrm{Y}}_{L L M}$ is a vector spherical harmonic defined by $\overrightarrow{\mathrm{Y}}_{L L M}$ $=\overrightarrow{\mathrm{L}} \mathrm{Y}_{L}^{M} /[L(L+1)]^{1 / 2}$, and $g_{L}(x)$ is proportional to the spherical Bessel function, having the power series expansion

$$
\begin{aligned}
g_{L}(x) & =\frac{4 \pi(i x)^{L}}{(2 L+1) ! !}\left(1-\frac{x^{2}}{2(2 L+3)}+\cdots\right), \\
(2 L+1) ! ! & =1 \times 3 \times 5 \times \cdots \times(2 L+1) .
\end{aligned}
$$

One normally retains only the leading term of (4) since, except for $x$-ray transitions, the wavelength of the emitted light is much larger than the atomic dimensions, making $\omega r / c \ll 1$. Nevertheless, it will be necessary to retain the leading two terms in the present work to obtain all contributions to the leading term of the $M 1$-transition integral.

An arbitrary vector potential $\vec{A}$, at the position of the $j$ th electron may be expanded in terms of the above complete basis set as

$$
\overrightarrow{\mathrm{A}}_{j}=\sum_{\omega L M}\left[c(\omega L M) \overrightarrow{\boldsymbol{Q}}_{\omega L M}(j)+c^{*}(\omega L M) \overrightarrow{\boldsymbol{Q}}_{\omega L M}^{*}(j)\right],
$$

where the expansion coefficients $c(\omega L M)$ and $c^{*}(\omega L M)$ are interpreted, respectively, as photon-annihilation and photon-creation operators in the theory of the quantized electromagnetic field. The spontaneous emission of a single photon by an $\mathrm{N}$-electron atom is due to the first-order coupling between the atom and the radiation field through the component $\sum_{j=1}^{N} \vec{a}_{\omega L M}^{*}(j)$ of the total vector potential associated with the creation operator.

We will consider in particular two-electron atoms in an electromagnetic field specified by the nuclear Coulomb scalar potential $V_{j}=-Z e / r_{j}$ and the photon vector potential $\vec{A}_{j}=\vec{a}_{\omega L N}^{*}(j)$. In the Dirac 16-component formalism, the stationary states satisfy ${ }^{14}$ [through terms of $O\left(\alpha^{2}\right)$ ]

$$
\begin{array}{r}
\left\{E-e V-\sum_{j=1,2}\left[m c^{2} \beta_{j}+\vec{\alpha}_{j} \cdot\left(c \overrightarrow{\mathrm{p}}_{j}-e \overrightarrow{\mathrm{A}}_{j}\right)\right]\right. \\
\left.+B_{12}-\Omega_{H}\right\} \Psi=0,
\end{array}
$$

where

$$
V=-Z e / r_{1}-Z e / r_{2}+e / r_{12},
$$




$$
\begin{aligned}
& B_{12}=\left(e^{2} / 2 r_{12}\right)\left(\vec{\alpha}_{1} \cdot \vec{\alpha}_{2}+\vec{\alpha}_{1} \cdot \vec{r}_{12} \vec{\alpha}_{2} \cdot \vec{r}_{12} / r_{12}^{2}\right),(8) \\
& \Omega_{H}=\mu_{B}\left(\alpha / 2 \pi-2.97 \alpha^{2} / \pi^{2}\right) \sum_{j=1,2} \vec{\sigma}_{j} \cdot \overrightarrow{\mathcal{H}}_{j} \\
& \vec{r}_{12}=\vec{r}_{1}-\vec{r}_{2}
\end{aligned}
$$

$\vec{\sigma}$ is the $4 \times 4$ Pauli spin matrix, $\mu_{B}=e \hbar / 2 m c=$ Bohr magneton, and $\overrightarrow{\mathcal{K}}_{j}=\nabla, \times \overrightarrow{\mathrm{A}}_{j}$ is the magnetic field. $E$ is the total energy including the electron rest-mass energy $2 m c^{2}, \beta_{1}$ and $\vec{\alpha}_{\text {, }}$ are the usual Dirac operators, $\bar{p}_{j}$ is the momentum operator, $\alpha=e^{2} / \hbar c$ is the fine-structure constant, $B_{12}$ is the Breit interaction operator, and $\Omega_{H}$ is that part of the quantum electrodynamic self-energy correction which is linear in $\overrightarrow{\nVdash C} .{ }^{15}$ As will be seen later, the contribution from $\Omega_{H}$ is $O(\alpha)$ relative to the terms in the $M 1$ transition integral evaluated in this paper and is neglected. Radiative corrections to Coulomb's law could also be included in Eq. (7), but again the contributions to the transition integral are smaller by a factor of $\alpha$.

The magnetic multipole transition moment is then proportional to the matrix element of the perturbation operator $-e \vec{\alpha} \cdot \vec{a}_{\omega L M}^{*}$ between the initial and final states. Specifically, Akhiezer and Berestetskii ${ }^{9}$ show that the emission probability for a photon in a state of magnetic type of angular $L$, component $M$, and parity $(-1)^{L+1}$ is

$$
A_{i \rightarrow f}=\frac{2(L+1)}{\hbar L(2 L+1)[(2 L-1) ! !]^{2}}\left(\frac{\omega}{c}\right)^{2 L+1}\left|\left(q_{L M}\right)_{i, f}\right|^{2} \text {, }
$$

where

$$
\begin{aligned}
{\left[a_{L M}(j)\right]_{i, f}=} & i e\left(\frac{i c}{\omega}\right)^{L}\left(\frac{L(2 L+1)}{4 \pi(L+1)}\right)^{1 / 2}(2 L-1) ! ! \\
& \times \int \Psi_{f}^{*} \vec{\alpha}_{j} \cdot \overrightarrow{\mathrm{a}}_{L M} * \Psi_{i} d r,
\end{aligned}
$$

$\omega$ is the transition frequency, and $\Psi_{i}$ and $\Psi_{f}$ are the initial- and final-state Dirac wave functions (omitting time-dependent factors). For an $\mathrm{N}$-electron atom, $q_{L N}$ is replaced in lowest order by $Q_{L M}=\sum_{j=1}^{N} q_{L M}(j)$.

Using hydrogenic Dirac wave functions ${ }^{14}$ to evaluate (11), the $M 12 s-1 s$ transition integral is

$$
\left(q_{10}\right)_{2 s, 1 s}=-\alpha^{3} e a_{0} Z^{2}\left[\frac{2}{27} \sqrt{2}-\frac{2}{81} \sqrt{2}+O(\alpha)+O\left(\alpha^{2} Z^{2}\right)\right],
$$

where $a_{0}$ is the Bohr radius. The first term is the relativistic energy correction arising from the expansion of the large and small components of the wave function in powers of $(\alpha Z)^{2}$ calculated by Breit and Teller. ${ }^{2}$ The second is a finite-wavelength correction from the expansion (4) of $g_{L}(\omega r / c)$ and has not been previously included. Substituting (12) into (10), the emission probability for oneelectron ions is

$$
A\left(2 s^{2} S-1 s^{2} S\right)=\hbar^{-1} 4(\omega / c)^{3}\left|\left(q_{10}\right)_{2 s, 1 s}\right|^{2}
$$

$$
=Z^{10} \alpha^{9} / 972 \times\left(2.4189 \times 10^{-17} \mathrm{sec}\right)^{-1},
$$

and for two-electron ions, in the limit of large $Z$,

$$
A\left(1 s 2 s^{3} S \rightarrow 1 s^{2}{ }^{1} S\right)=\frac{2}{3} A\left(2 s^{2} S \rightarrow 1 s^{2} S\right)+O\left(\alpha^{9} Z^{9}\right)
$$

summed over final states and averaged over initial states. The factor of $\frac{2}{3}$ is the ratio of statistical weights for the $2 s^{2} S$ and $1 s 2 s^{3} S$ states. The hydrogenic approximation (14) to the emission probability for two-electron ions results from neglecting the electron-mutual-interaction terms $e^{2} / r_{12}+B_{12}$ in (6) and is of low accuracy. The effects of the mutualinteraction terms are more easily evaluated within the Pauli approximation as developed in Sec. III.

\section{MAGNETIC DIPOLE TRANSITION MOMENT IN PAULI APPROXIMATION}

The usual definition of the magnetic dipole moment operator in the Pauli approximation is

$$
\vec{Q}_{1}=\sum_{j} \mu_{B}\left(\vec{L}_{j}+\vec{\sigma}_{j}\right),
$$

where $\vec{L}_{j}$ is the angular momentum operator for the $j$ th electron and $\vec{\sigma}_{j}$ is the Pauli spin operator. Since matrix elements of (15) varish for $n l-n^{\prime} l$ transitions with $n \neq n^{\prime}$, it is necessary to obtain the correction terms $O\left(\alpha^{2} Z^{2}\right)$ with respect to (15). The $O\left(\alpha^{2} Z^{2}\right)$ corrections to the expectation value of the magnetic moment operator in the $1 s 2 s^{3} S$ state have been evaluated by Perl and Hughes, ${ }^{16}$ assuming a constant, uniform magnetic field. The operator contains additional terms when one considers transition integrals since finite-wavelength effects are important.

The general procedure for obtaining relativistic corrections in the Pauli approximation is to eliminate the contribution from the "small" components of the wave functions to successively higher orders in $\alpha^{2} Z^{2}$ by repeated application of the unitary Foldy Wouthuysen (FW) ${ }^{17}$ transformation, as described also by Messiah. ${ }^{18}$ However, Messiah's formulas are applicable only to time-independent potentials and must be modified since the photon vector potential $\vec{A}_{j}$ is in fact time dependent. The Hamiltonian $H$ which governs the evolution of states in the Pauli representation is of the form

$$
H=U H_{D} U^{\dagger}-\frac{i U \partial U^{\dagger}}{\partial t}
$$

where $U$ is the FW transformation operator and $H_{D}$ is the Dirac Hamiltonian. The term involving $-i \partial / \partial t$ vanishes if the external field is time independent.

Writing

$$
\overrightarrow{\mathrm{A}}_{j}=\overrightarrow{\mathrm{a}}_{L M}^{*}(j) e^{i \omega t}
$$

and 


$$
\vec{\pi}_{j}=\overrightarrow{\mathrm{p}}_{j}-e \overrightarrow{\mathrm{A}}_{j} / c,
$$

the effective two-electron Hamiltonian in the second Pauli approximation obtained from a multiple FW transformation is

$$
H=H(1)+H(2)+B_{12}^{\prime},
$$

where

$$
\begin{array}{r}
\left.H(j)=\frac{\left(\vec{\sigma}_{j} \cdot \vec{\pi}_{j}\right)^{2}}{2 m}-\frac{1}{8 m^{2} c^{2}}\left[\left(\vec{\sigma}_{j} \cdot \vec{\pi}_{j}\right),\left(\vec{\sigma}_{j} \cdot \vec{\pi}_{j}\right), e V-i \frac{\partial}{\partial \imath}\right)\right] \\
+\frac{\left(\vec{\sigma}_{j} \cdot \vec{\pi}_{j}\right)^{4}}{8 m^{3} c^{3}}
\end{array}
$$

and

$$
\begin{aligned}
B_{12}^{\prime}= & -\frac{e^{2}}{2 m^{2} c^{2} r_{12}}\left(\vec{\pi}_{1} \cdot \vec{\pi}_{2}+\frac{\overrightarrow{\mathrm{r}}_{12} \cdot\left(\overrightarrow{\mathrm{r}}_{12} \cdot \vec{\pi}_{1}\right) \vec{\pi}_{2}}{r_{12}^{2}}\right) \\
& +\frac{\mu_{B} e}{m c r_{12}^{3}}\left(\overrightarrow{\mathrm{r}}_{12} \times \vec{\pi}_{2} \cdot \vec{\sigma}_{1}+\overrightarrow{\mathrm{r}}_{21} \times \vec{\pi}_{1} \cdot \vec{\sigma}_{2}\right) \\
& +\mu_{B}^{2}\left[-\frac{8}{3} \pi\left(\vec{\sigma}_{1} \cdot \vec{\sigma}_{2}\right) \delta^{(3)}\left(\overrightarrow{\mathrm{r}}_{12}\right)\right. \\
& \left.+\frac{1}{r_{12}^{3}}\left(\vec{\sigma}_{1} \cdot \vec{\sigma}_{2}-\frac{3\left(\vec{\sigma}_{1} \cdot \overrightarrow{\mathrm{r}}_{12}\right)\left(\vec{\sigma}_{2} \cdot \overrightarrow{\mathrm{r}}_{12}\right)}{r_{12}^{2}}\right)\right] .
\end{aligned}
$$

The three terms of (21) in brackets are, respectively, the mutual electron orbit-orbit, spin-orbit, and spin-spin interactions arising from the $B_{12}$ term of (6). $H$ is defined such that the Pauli eigenfunctions may be taken as orthonormal up to, but not including, terms of $O\left(\alpha^{4} Z^{4}\right)$.

The perturbation operator responsible for $M 1$ transitions is obtained by separating out of (20) and (21) the terms linear in $\vec{A}$. Making use of the relations

$$
\begin{aligned}
(\vec{\sigma} \cdot \overrightarrow{\mathrm{A}})(\vec{\sigma} \cdot \overrightarrow{\mathrm{B}}) & =\overrightarrow{\mathrm{A}} \cdot \overrightarrow{\mathrm{B}}+\overrightarrow{i \sigma} \cdot \overrightarrow{\mathrm{A}} \times \overrightarrow{\mathrm{B}}, \\
(\vec{\sigma} \cdot \vec{\pi})^{2} & =\vec{\pi}^{2}-e \vec{\sigma} \cdot \overrightarrow{\mathfrak{C}} / c, \\
(\vec{\sigma} \cdot \vec{\pi})^{4} & =\left(\vec{\pi}^{2}-e \vec{\sigma} \cdot \overrightarrow{\mathfrak{H}} / c\right)^{2}, \\
{[(\vec{\sigma} \cdot \vec{\pi}),[(\vec{\sigma} \cdot \vec{\pi}), V]] } & =-\nabla^{2} V-2 \vec{\sigma} \cdot(\nabla V \times \vec{\pi}),
\end{aligned}
$$

and

$$
\begin{aligned}
& {\left[(\vec{\sigma} \cdot \vec{\pi}),\left[(\vec{\sigma} \cdot \vec{\pi}), i \frac{\partial}{\partial t}\right]\right]} \\
& \quad=(e / c) i \omega \nabla \cdot \overrightarrow{\mathrm{A}}-(e / c) \omega \overrightarrow{\mathcal{C}} \cdot \vec{\sigma}+(2 e / c) i \omega \overrightarrow{\mathrm{A}} \times \overrightarrow{\mathrm{p}},
\end{aligned}
$$

the linear interaction operator is

$$
H_{\mathrm{int}}=H_{\mathrm{int}}(1)+H_{\mathrm{int}}(2)+H_{\mathrm{int}}(12),
$$

where

$$
\begin{aligned}
H_{\mathrm{int}}(j)= & -(e / 2 m c)\left(\overrightarrow{\mathrm{p}}_{j} \cdot \overrightarrow{\mathrm{A}}_{j}+\overrightarrow{\mathrm{A}}_{j} \cdot \overrightarrow{\mathrm{p}}_{j}\right)-(e / 2 m c) \vec{\sigma}_{j} \cdot \overrightarrow{\mathcal{K}}_{j}+\left(e / 8 m^{3} c^{3}\right)\left[\overrightarrow{\mathrm{p}}_{j}^{2},\left(\overrightarrow{\mathrm{p}}_{j} \cdot \overrightarrow{\mathrm{A}}_{j}+\overrightarrow{\mathrm{A}}_{j} \cdot \overrightarrow{\mathrm{p}}_{j}\right)\right]_{+} \\
& +\left(e / 8 m^{3} c^{3}\right)\left[p_{j}^{2}, \vec{\sigma}_{j} \cdot \overrightarrow{\mathcal{H}}_{j}\right]_{+}-\left(e^{2} / 4 m^{2} c^{3}\right) \vec{\sigma}_{j} \cdot \nabla j \times \overrightarrow{\mathrm{A}}_{j}+\left(e \omega / 8 m^{2} c^{3}\right)\left(i \nabla_{j} \cdot \overrightarrow{\mathrm{A}}_{j}-\vec{\sigma}_{j} \cdot \overrightarrow{\mathcal{H}}_{j}+2 i \vec{\sigma}_{j} \cdot \overrightarrow{\mathrm{A}}_{j} \times \overrightarrow{\mathrm{p}}_{j}\right) \\
H_{\mathrm{int}}(12)= & \frac{e^{3}}{2 m^{2} c^{3} r_{12}}\left(\overrightarrow{\mathrm{A}}_{1} \cdot \overrightarrow{\mathrm{p}}_{2}+\overrightarrow{\mathrm{p}}_{1} \cdot \overrightarrow{\mathrm{A}}_{2}+\frac{\left(\overrightarrow{\mathrm{r}}_{12} \cdot \overrightarrow{\mathrm{A}}_{1}\right)\left(\overrightarrow{\mathrm{r}}_{12} \cdot \overrightarrow{\mathrm{p}}_{2}\right)+\left(\overrightarrow{\mathrm{r}}_{12} \cdot \overrightarrow{\mathrm{A}}_{2}\right)\left(\overrightarrow{\mathrm{r}}_{12} \cdot \overrightarrow{\mathrm{p}}_{1}\right)}{r_{12}^{2}}\right) \\
& -\left(\mu_{B} e^{2} / m c^{2} r_{12}^{3}\right)\left(\overrightarrow{\mathrm{r}}_{12} \times \overrightarrow{\mathrm{A}}_{2} \cdot \vec{\sigma}_{1}+\overrightarrow{\mathrm{r}}_{21} \times \overrightarrow{\mathrm{A}}_{1} \cdot \vec{\sigma}_{2}\right) .
\end{aligned}
$$

The notation $[,]_{+}$denotes the anticommutator.

For the $L=1$ case we have

$$
\overrightarrow{\mathrm{A}}_{j}=(1 / \sqrt{ } 2)\left[g_{1}\left(\omega r_{j} / c\right) \overrightarrow{\mathrm{L}}_{j} Y_{1}^{M}\left(\theta_{j}, \varphi_{j}\right)\right]^{*},
$$

and, from (11), $Q_{1 \mathrm{M}}=-(c / \omega)\left(\frac{3}{8} \pi\right)^{1 / 2} H_{\mathrm{int}}$. Using (28) and (29), the expression for the vector magnetic dipole moment operator becomes

$$
\begin{aligned}
\overrightarrow{\mathrm{Q}}_{1} & =\mu_{B} \sum_{j=1,2}\left(\overrightarrow{\mathrm{L}}_{j}+\vec{\sigma}_{j}-\frac{p_{j}^{2}}{2 m^{2} c^{2}}\left(\overrightarrow{\mathrm{L}}_{j}+\vec{\sigma}_{j}\right)+\frac{1}{4 m^{2} c^{2}} \overrightarrow{\mathrm{p}}_{j} \times\left(\overrightarrow{\mathrm{p}}_{j} \times \vec{\sigma}_{j}\right)+\frac{1}{10}(\omega / c)^{2}\left[\left(\overrightarrow{\mathrm{r}}_{j} \times\left(\overrightarrow{\mathrm{r}}_{j} \times \vec{\sigma}_{j}\right)-r_{j}^{2}\left(\overrightarrow{\mathrm{L}}_{j}+\vec{\sigma}_{j}\right)\right]\right.\right. \\
& \left.-\frac{Z e^{2}}{2 m c^{2} r_{j}^{3}}\left[\overrightarrow{\mathrm{r}}_{j} \times\left(\overrightarrow{\mathrm{r}}_{j} \times \vec{\sigma}_{j}\right)\right]+\frac{\omega}{4 m c^{2}} \vec{\sigma}_{j}\right)+\frac{\mu_{B} e^{2}}{2 m c^{2} r_{12}}\left(\frac{\overrightarrow{\mathrm{r}}_{12} \times\left[\overrightarrow{\mathrm{r}}_{12} \times\left(\vec{\sigma}_{1}+\vec{\sigma}_{2}\right)\right]+\left(\overrightarrow{\mathrm{r}}_{1} \times \overrightarrow{\mathrm{r}}_{2}\right) \overrightarrow{\mathrm{r}}_{12} \cdot\left(\overrightarrow{\mathrm{p}}_{1}+\overrightarrow{\mathrm{p}}_{2}\right)}{r_{12}^{2}}-\left(\overrightarrow{\mathrm{r}}_{1} \times \overrightarrow{\mathrm{p}}_{2}\right)-\left(\overrightarrow{\mathrm{r}}_{2} \times \overrightarrow{\mathrm{p}}_{1}\right)\right) .
\end{aligned}
$$

In the above we have used (to the order required)

$$
\begin{aligned}
\vec{\sigma} \cdot \overrightarrow{\mathcal{H}} & =K\left[\vec{\sigma}+\frac{1}{10}(\omega / c)^{2}\left(\overrightarrow{\mathrm{r}} \times(\overrightarrow{\mathrm{r}} \times \vec{\sigma})-r^{2} \vec{\sigma}\right)\right]_{M}, \\
\overrightarrow{\mathrm{p}} \cdot \overrightarrow{\mathrm{A}} & =K\left[\overrightarrow{\mathrm{L}}-\frac{1}{10}(\omega / c)^{2} r^{2} \overrightarrow{\mathrm{L}}\right]_{M}, \\
\vec{\sigma} \cdot \overrightarrow{\mathrm{r}} \times \overrightarrow{\mathrm{A}} & =K[\overrightarrow{\mathrm{r}} \times(\overrightarrow{\mathrm{r}} \times \vec{\sigma})]_{M},
\end{aligned}
$$

and

$$
\begin{aligned}
i \omega\langle & \left.f\left|\vec{\sigma}_{j} \cdot \overrightarrow{\mathrm{A}}_{j} \times \overrightarrow{\mathrm{p}}_{j}\right| i\right\rangle=i\left\langle f\left|\left[\vec{\sigma}_{j} \cdot \overrightarrow{\mathrm{A}}_{j} \times \overrightarrow{\mathrm{p}}_{j}, H_{\mathrm{NR}}\right]\right| i\right\rangle \\
= & K\left(f \mid \overrightarrow{\mathrm{p}}_{j} \times\left(\overrightarrow{\mathrm{p}}_{j} \times \vec{\sigma}_{j}\right)-\left(Z e^{2} / r_{j}^{3}\right)\left[\overrightarrow{\mathrm{r}}_{j} \times\left(\overrightarrow{\mathrm{r}}_{j} \times \sigma_{j}\right)\right]\right. \\
& +\left(e^{2} / r_{12}^{3}\right)\left[\overrightarrow{\mathrm{r}}_{12} \times\left(\overrightarrow{\mathrm{r}}_{12} \times \vec{\sigma}_{j}\right)\right]|i\rangle_{M}
\end{aligned}
$$


for $j=1$, 2, where $K=(\omega / c)\left(\frac{8}{3} \pi\right)^{1 / 2}$ and the notation $[\overrightarrow{\mathrm{R}}]_{M}$ denotes the tensor components $\mp\left(R_{x} \pm R_{y}\right) / \sqrt{2}$ or $R_{z}$ for $M= \pm 1$ or 0 , respectively. In view of the commutator relation (34), the operator (30) is correct only when applied to eigenfunctions of the nonrelativistic Hamiltonian $H_{\mathrm{NR}}$.

For the $2{ }^{3} S-1{ }^{1} S$ transition of helium, the spinindependent terms and terms symmetric in the spin ope rators do not contribute because of the orthogonality of the initial and final spin states. The $M=0$ component of the magnetic dipole transition matrix element then reduces to

$$
\begin{aligned}
\left\langle{ }^{1} S\left|Q_{10}\right|{ }^{3} S\right\rangle= & \mu_{B}\left\langle 1^{1} S\right|-\left(2 / 3 m^{2} c^{2}\right)\left(p_{1}^{2}-p_{2}^{2}\right) \\
& -\frac{1}{6}(\omega / c)^{2}\left(r_{1}^{2}-r_{2}^{2}\right) \\
& +\left(Z e^{2} / 3 m c^{2}\right)\left(r_{1}^{-1}-r_{2}^{-1}\right)\left|2^{3} S\right\rangle,
\end{aligned}
$$

omitting the spin parts of the wave functions. The terms in (35) represent a mixture of finite-wavelength and relativistic energy corrections to the magnetic dipole moment operator. ${ }^{19}$

In the limit of large $Z,(35)$ is related to the hydrogenic transition integral by

$$
\left\langle 1^{1} S\left|Q_{10}\right| 2^{3} S\right\rangle \sim \sqrt{2}\left\langle 1 s\left|q_{10}\right| 2 s\right\rangle,
$$

where

$$
q_{10}=\mu_{B}\left[-2 p^{2} / 3 m^{2} c^{2}-\frac{1}{B}(\omega r / c)^{2}+Z e^{2} / 3 m c^{2} r\right] \sigma_{z} .
$$

Evaluation of $\left\langle 1 s\left|q_{10}\right| 2 s\right\rangle$ with nonrelativistic hydrogenic wave functions yields $-\alpha^{3} e a_{0} Z^{2}\left(\frac{2}{27} \sqrt{2}-\frac{2}{81} \sqrt{2}\right)$, in agreement with the result (12) obtained by expanding the exact Dirac wave functions in powers of $\alpha^{2} Z^{2}$. From (13), (14), and (36), the averaged emission probability from the $2{ }^{3} S$ state of helium is

$$
A\left(2{ }^{3} S \rightarrow 1^{1} S\right)=\hbar^{-1} \frac{4}{3}(\omega / c)^{3}\left|\left\langle 1^{1} S\left|Q_{10}\right| 2^{3} S\right\rangle\right|^{2} .
$$

(See Note added in proof.)

\section{NUMERICAL RESULTS FOR TWO-ELECTRON SYSTEMS}

We wish to obtain accurate solutions of

$$
\left(H_{\mathrm{NR}}-W\right) \psi=0
$$

for the $2^{3} S$ and $1^{1} S$ states of heliumlike ions, where

$$
\begin{aligned}
& H_{\mathrm{NR}}=H_{0}+Z^{-1} V_{12}, \\
& H_{0}=-\frac{1}{2} \nabla_{1}^{2}-\frac{1}{2} \nabla_{2}^{2}-r_{1}^{-1}-r_{2}^{-1}, \\
& V_{12}=r_{12}^{-1} .
\end{aligned}
$$

In the above, the unit of energy is $Z^{2}$ a. u. and the unit of length is $Z^{-1} a_{0}$. An efficient method of generating accurate solutions for the entire helium isoelectronic sequences is provided by the varia- tion-perturbation procedure of Scherr and Knight. ${ }^{2}$ Introducing the $Z^{-1}$ expansin:is

$$
\begin{gathered}
\psi=\psi^{(0)}+\sum_{n=1}^{\infty} \psi^{(n)} Z^{-n}, \\
W=W^{(0)}-\sum_{i=1}^{\infty} W^{(n)} Z^{-n},
\end{gathered}
$$

the zero-order function satisfies the hydrogenic equation

$$
\left(H_{0}-W^{(0)}\right) \psi^{(0)}=0,
$$

and the $\psi^{(n)}, n>0$, satisfy a series of perturbation equations which are often solved by finding the stationary values of the functionals

$$
\begin{aligned}
J_{n}= & \left\langle\psi^{(n)}\left|H_{0}-W^{(0)}\right| \psi^{(n)}\right\rangle+2\left\langle\psi^{(n)}\left|V_{12}-W^{(1)}\right| \psi^{(n-1)}\right\rangle \\
& -2 \sum_{r=0}^{2 n-1} W^{(r)} \sum_{q=n-r}^{r}\left\langle\psi^{(q)} \mid \psi^{(2 n-r-q)}\right\rangle
\end{aligned}
$$

with respect to arbitrary variations of $\psi^{(n)}$, assuming that all the $\psi^{(m)}$ with $m<n$ are known exactly.

In the formulation of Dalgarno and Drake, ${ }^{12} \mathrm{a}$ set of $M$ orthonormal functions $\varphi_{i}$ is introduced, each of which is constructed from a linear combination of $M$ functions of the form

$$
\chi_{j}=\left(1 \pm P_{12}\right) r_{1}^{a} r_{2}^{b} r_{12}^{c} e-\alpha^{\prime} r_{1}-\beta r_{2}
$$

and satisfies

$$
\left\langle\varphi_{i}\left|H_{0}\right| \varphi_{j}\right\rangle=\epsilon_{i} \delta_{i, j} .
$$

Here, $P_{12}$ indicates the exchange of the labels 1 and 2 , with the + sign referring to the $1^{1} S$ state and the - sign referring to the $2{ }^{3} S$ state. The scale factors $\alpha$ and $\beta$ are set equal to their hydrogenic values, i. e., $\alpha=\beta=1$ for the $1^{1} S$ state and $\alpha=2 \beta=1$ for the $2^{3} S$ state. Then one of the $\varphi_{i}$, say $\varphi_{s}$, is the exact hydrogenic $\psi^{(0)}$ with $\epsilon_{i}=W^{(0)}$, and the remaining basis functions form a synthetic representation of the excited states of $H_{0}$ which, together with $\varphi_{s}$, form a complete set. If we expand $\psi^{(n)}$ in the finite basis set $\varphi_{i}$,

$$
\psi^{(n)}=\sum_{i \neq s}^{M} a_{i}^{(n)} \varphi_{i},
$$

and evaluate $J_{n}$, demanding that its value be stationary with respect to variations of the $a_{i}^{(n)}$, it follows that $\psi^{(n)}$ and $W^{(n)}$ are determined by the recursion relations ${ }^{12}$

$$
\begin{gathered}
\psi^{(n)}=\sum_{i \neq s}^{n}\left(\frac{\left\langle\psi^{(n-1)}\left|V_{12}\right| \varphi_{i}\right\rangle}{\epsilon_{s}-\epsilon_{i}}-\sum_{p=1}^{n=1} \frac{W^{(p)}\left\langle\psi^{(n-p)} \mid \varphi_{i}\right\rangle}{\epsilon_{s}-\epsilon_{i}}\right) \varphi_{i}, \\
W^{(n)}=\left\langle\psi^{(n-1)}\left|V_{12}\right| \psi^{(0)}\right\rangle-\sum_{p=1}^{n-1} W^{(p)}\left\langle\psi^{(n-p)} \mid \psi^{(0)}\right\rangle .
\end{gathered}
$$

Since the $\psi^{(m)}, m<n$ must be known exactly, the above recursion relations lead to the exact solutions only in the limit of a complete, infinitedimensional basis set. However, accurate results 
TABLE I. $1 s^{2}{ }^{1} S-1 s 2 s{ }^{3} S$ transition-integral expansion coefficients, ${ }^{\mathbf{a}}$

\begin{tabular}{cccc}
\hline \hline Order & $p_{1}^{2}-p_{2}^{2}$ & $r_{1}^{2}-r_{2}^{2}$ & $r_{1}^{-1}-r_{2}^{-1}$ \\
\hline 0 & 0.592593 & -4.213992 & 0.296296 \\
1 & -0.29569 & -4.2602 & -0.02147 \\
2 & 0.00625 & -3.1229 & -0.02211 \\
3 & -0.03400 & -2.0631 & -0.02498 \\
4 & -0.03206 & -1.3264 & -0.02422 \\
5 & -0.02911 & -0.8300 & -0.02256 \\
6 & -0.02595 & -0.4796 & -0.02059 \\
7 & -0.02292 & -0.1756 & -0.01869 \\
8 & -0.02119 & 0.1903 & -0.01748 \\
9 & -0.02039 & 0.6563 & -0.01716 \\
\hline \hline
\end{tabular}

${ }^{2}$ The matrix element of an operator $\theta$ for an ion with nuclear charge $Z$ is $\left\langle 1 s^{2}{ }^{1} s|\theta| 1 s 2 s^{3} S\right\rangle=\theta_{0}+\theta_{1} Z^{-1}+\theta_{2} Z^{-2}$ $+\cdots+\theta_{9} Z^{-9}$, where the unit of length is $Z^{-1} a_{0}$.

are still obtainable in a finite basis set, with the additional advantage that only a single matrix diagonalization of $H_{0}$ is required [cf. Eq. (48)] to generate results for the entire isoelectronic sequence.

The above $Z^{-1}$ expanded wave functions were used to calculate the coefficients in the $Z^{-1}$ expansions of the matrix elements $\left\langle 1^{1} S\left|p_{1}^{2}-p_{2}^{2}\right| 2^{3} S\right\rangle$, $\left\langle 1^{1} S\left|r_{1}^{2}-r_{2}^{2}\right| 2^{3} S\right\rangle$, and $\left\langle 1^{1} S\left|r_{1}^{-1}-r_{2}^{-1}\right| 2^{3} S\right\rangle$. Both the initial- and final-state wave functions were constructed from the 47 basis functions of the form (47) having $a+b+c \leq 6$ and $c \leq 4$. The resulting matrix-element expansion coefficients are given in Table I, and the summed matrix elements and $M 1$ decay rates for the ions up to Fe xxv are given in Table II. The expansions summed through ninth order are sufficient to determine the matrix elements for neutral helium to three or four significant figures. For example, the $Z=2$ values from Table II, $0.43844,-7.4984$, and 0.27412 , compare well with the values $0.43835,-7.5022$, and 0.27406 calculated directly from 50 -term correlated variational wave functions constructed from linear combinations of terms of the form (47), but with $\alpha$ and $\beta$ chosen to optimize the energy.

\section{DISCUSSION}

The He I $M 1$ decay rate, $1.27 \times 10^{-4} \mathrm{sec}^{-1}$, is a factor of 3 larger than Griem's estimate. ${ }^{8}$ However, Griem's estimates have an incorrect $Z^{8}$ dependence, causing the disagreement with his data to increase with increasing $Z .^{21}$ The correct $Z^{10}$ dependence for the $M 1$ decay rate has also been derived by Freeman et al. ${ }^{22}$

The Ar xvir mean life (212.7 nsec) lies above the

TABLE II. $1 s 2 s^{3} S-1 s^{2}{ }^{1} S$ energy differences, transition integrals, ${ }^{a}$ and $M 1$ decay rates for the heliumlike ions.

\begin{tabular}{|c|c|c|c|c|c|}
\hline$Z$ & $\Delta E(\text { a. u. })^{\mathrm{b}}$ & $p^{2} / Z^{2}$ & $Z^{2} r^{2}$ & $1 /(Z r)$ & $A_{i-f}\left(\sec ^{-1}\right)$ \\
\hline 2 & 0.72850 & 0.43844 & -7.4984 & 0.27412 & $1.272 \times 10^{-4}$ \\
\hline 3 & 2.16918 & 0.49290 & -6.0779 & 0.28532 & $2.039 \times 10^{-2}$ \\
\hline 4 & 4.35840 & 0.51837 & -5.5126 & 0.28903 & $5.618 \times 10^{-1}$ \\
\hline 5 & 7.29707 & 0.53337 & -5.2099 & 0.29087 & $6.695 \times 10^{0}$ \\
\hline 6 & 10.98549 & 0.54330 & -5.0215 & 0.29197 & $4.856 \times 10^{1}$ \\
\hline 7 & 15.42376 & 0.55036 & -4.8929 & 0.29269 & $2.532 \times 10^{2}$ \\
\hline 8 & 20.61194 & 0.55565 & -4.7997 & 0.29321 & $1.044 \times 10^{3}$ \\
\hline 9 & 26.55007 & 0.55976 & -4.7289 & 0.29360 & $3.608 \times 10^{3}$ \\
\hline 10 & 33.23815 & 0.56305 & -4.6734 & 0.29390 & $1.087 \times 10^{4}$ \\
\hline 11 & 40.67621 & 0.56574 & -4.6287 & 0.29414 & $2.935 \times 10^{4}$ \\
\hline 12 & 48.86425 & 0.56797 & -4.5920 & 0.29434 & $7.243 \times 10^{4}$ \\
\hline 13 & 57.80226 & 0.56987 & -4.5612 & 0.29450 & $1.658 \times 10^{5}$ \\
\hline 14 & 67.49027 & 0.57149 & -4.5350 & 0.29464 & $3.563 \times 10^{5}$ \\
\hline 15 & 77.92826 & 0.57290 & -4.5125 & 0.29476 & $7.251 \times 10^{5}$ \\
\hline 16 & 89.11625 & 0.57413 & -4.4930 & 0.29486 & $1.408 \times 10^{6}$ \\
\hline 17 & 101.0542 & 0.57521 & -4.4758 & 0.29495 & $2.622 \times 10^{6}$ \\
\hline 18 & 113.7422 & 0.57618 & -4.4607 & 0.29503 & $4.709 \times 10^{6}$ \\
\hline 19 & 127.1802 & 0.57704 & -4.4472 & 0.29510 & $8.187 \times 10^{6}$ \\
\hline 20 & 141.3681 & 0.57782 & -4.4351 & 0.29516 & $1.383 \times 10^{7}$ \\
\hline 21 & 156.3061 & 0.57852 & -4.4242 & 0.29522 & $2.275 \times 10^{7}$ \\
\hline 22 & 171.9941 & 0.57916 & -4.4143 & 0.29527 & $3.656 \times 10^{7}$ \\
\hline 23 & 188.4320 & 0.57975 & -4.4053 & 0.29532 & $5.751 \times 10^{?}$ \\
\hline 24 & 205.6200 & 0.58028 & -4.3971 & 0.29536 & $8.870 \times 10^{7}$ \\
\hline 25 & 223.5579 & 0.58077 & -4.3895 & 0.29540 & 1. $344 \times 10^{8}$ \\
\hline 26 & 242.2459 & 0.58123 & -4.3826 & 0.29544 & $2.002 \times 10^{8}$ \\
\hline
\end{tabular}

${ }^{a}$ An operator $\mathrm{O}$ is understood to mean $\mathrm{O}_{1}-\mathrm{O}_{2}$, with the spin parts of the wave functions omitted when calculating matrix elements. Atomic units are used except as noted.

${ }^{b}$ Nonrelativistic energy differences are used throughout. Relativistic corrections increase the $A_{i \sim f}$ values by less than 1 or $2 \%$ for $Z \leqslant 20$. 
quoted error limit in Schmieder and Marrus's ${ }^{12}$ experimental value, $172 \pm 30 \mathrm{nsec}$ ( $95 \%$ confidence). Relativistic corrections to the $2{ }^{3} S-1{ }^{1} S$ transition frequency $(\omega)$ decrease the theoretical mean life only by about $1 \%$ and cannot explain the discrepancy. Also, the neglected terms in the transition integral are of $O(\alpha)$ and $O\left(\alpha^{2} Z^{2}\right)$ relative to the leading term and should not change the results by more than 1 or $2 \%$. The discrepancy, if real, could be explained by an additional mechanism depopulating the metastable states.

Freeman et al. ${ }^{22}$ have derived the $M 1$ decay rate of $\mathrm{C} \vee$ from observed coronal line intensities. Their value $\left(37 \mathrm{sec}^{-1}\right)$ is in satisfactory agreement with $48.6 \mathrm{sec}^{-1}$ given in Table II. The above authors deduced from their coronal observations the semiempirical formula $A_{i \bullet f}=4.4 \times 10^{9} \lambda^{-5} \mathrm{sec}^{-1}$ ( $\lambda$ in $\AA$ ) for the decay rates along the isoelectronic sequence. By chance, their formula comes remarkably close to the exact asymptotic relation $A_{i \rightarrow f}=4.4059 \times 10^{9} \lambda^{-5} \mathrm{sec}^{-1}$, where $\lambda$ is the hydrogenic wavelength $(\AA)$.

Note added in proof. It is assumed in this paper that, in the absence of interparticle interactions, the two-particle FW Hamiltonian is a sum of oneparticle Hamiltonians. R. A. Krajcik and L. L. Foldy [ Phys. Rev. Letters 24, 545 (1970)] have recently found some additional nonadditive terms arising from a transformation associated with center-of-mass motion. These terms, although of the right order in $\alpha$, can be shown to make no contribution to the magnetic dipole transition operator.

\section{ACKNOWLEDGMENTS}

The author expresses thanks to C. Schwartz for pointing out an error in the Pauli form of the magnetic dipole moment operator and for other helpful correspondence. Results similar to those presented herein have been obtained independently by him. Preprints were kindly sent prior to publication by R. W. Schmieder and R. Marrus on the Ar xvir lifetime measurement and by A. H. Gabriel on the solar coronal observations. Financial support by the National Research Council of Canada is gratefully acknowledged.

\section{APPENDIX: NUCLEAR-SPIN-INDUCED TRANSITIONS FROM $2^{3} s$ STATE}

The photon-emission probabilities for transitions of both the $M L$ and $E L$ types are determined by formulas of the form
$A_{M L}$ or $E L=\frac{2(L+1)}{L(2 L+1)[(2 L-1) ! !]^{2}}\left(\frac{\omega}{c}\right)^{2 L+1}\left|Q_{M L \text { or } E L}\right|^{2}$,

where $Q$ is the appropriate transition integral. $Q_{M L}$ is $O\left(\alpha Z^{-L+1}\right)$ and $Q_{E L}$ is $O\left(Z^{-L}\right)$ for allowed transitions, whereas the inhibited $2{ }^{3} S-1^{1} S M 1$ transition integral is $O\left(\alpha^{3} Z^{2}\right)$. Since ${ }^{1} D_{2}^{e}$ states connect directly with the ground state through an $E 2$ transition, it is necessary to estimate the nuclear-spin-induced mixing of ${ }^{1} D_{2}^{e}$ states with the $2{ }^{3} S_{1}$ state. $E 1$ transitions to the ground state need not be considered because the upper state is of odd parity. Since all the fine- and hyperfinestructure interactions are parity conserving, they mix the $2{ }^{3} S_{1}$ state only with states of even parity.

The hyperfine-structure Hamiltonian is ${ }^{23}$

$$
\begin{aligned}
H_{\mathrm{hPs}}= & -2 \mu_{B} \sum_{j=1,2}\left\{-\frac{4}{3} \pi\left(\vec{\sigma}_{j} \cdot \vec{\mu}_{I}\right) \delta^{(3)}\left(\overrightarrow{\mathrm{r}}_{j}\right)-\left(1 / r_{j}^{3}\right) L_{j} \cdot \vec{\mu}_{I}\right. \\
& \left.+\left(1 / 2 r_{j}^{3}\right)\left[\vec{\sigma}_{j} \cdot \vec{\mu}_{I}-3\left(\vec{\sigma}_{j} \cdot \overrightarrow{\mathrm{r}}_{j}\right)\left(\vec{\mu}_{I} \cdot \overrightarrow{\mathrm{r}}_{j}\right) / r_{j}^{2}\right]\right\}, \quad \text { (A2) }
\end{aligned}
$$

where $\vec{\mu}_{I}=-g_{I} \mu_{N} \vec{I}$ is the nuclear magnetic moment. $H_{\text {his }}$ may be thought of as a first-order perturbation operator mixing ${ }^{(2 S+1)} L$ states with the $2{ }^{3} S$ state with mixing coefficients

$$
\left\langle 2^{3} S\left|H_{\mathrm{hfs}}\right|^{(2 S+1)} L\right\rangle /\left[E\left(2^{3} S\right)-E\left({ }^{(2 S+1)} L\right)\right] .
$$

We need consider only the ${ }^{1} D_{2}^{e}$ states since no others connect directly with the ground state through an E2 transition.

The terms of (A2) are, respectively, the Fermi contact term, the nuclear-spin-electron-orbit term, and the nuclear-spin-electron-spin term. Only the nuclear-spin-electron-spin term is effective in connecting $S$ and $D$ states because the spatial part of the operator transforms as a spherical tensor of rank 2. ${ }^{24}$ The mixing coefficient $\left\langle 2^{3} S\left|H_{\mathrm{hfs}}\right| n^{1} D\right\rangle / \Delta E$ is then of $O\left(\alpha^{2} Z m / M\right)$, where $m$ and $M$ are the electron and nuclear masses, and we have included the scaling with the nuclear charge $Z$. From (A1), the nuclear-spin-induced $E 2$ emission probability is of $O\left(\alpha^{9} Z^{8} \mathrm{~m}^{2} / M^{2}\right)$, which is smaller than the $M 1$ emission probability by a factor of $O\left(m^{2} /(Z M)^{2}\right)$.

Similarly, the mixing of the $2^{3} S_{1}$ state with the final $1^{1} S_{0}$ state by the Fermi contact term allows the $2^{3} S-1{ }^{1} S M 1$ transition to proceed by the nuclearspin mixing path. ${ }^{25}$ Although the $2{ }^{3} S-2{ }^{3} S M 1$ transition integral is $O(\alpha)$, the over-all process, including the nuclear-spin mixing coefficient, is less probable than the direct $2{ }^{3} S-1{ }^{1} S M 1$ transition by a factor of $O\left(m^{2} /(Z M)^{2}\right)$.
${ }^{1}$ A. H. Gabriel and C. Jordan, Monthly Notices Roy. Astron. Soc. 145, 241 (1969).

${ }^{2}$ G. Breit and E. Teller, Astrophys. J. $\underline{91}, 215$ (1940).

${ }^{3}$ J. S. Mathis, Astrophys. J. $\underline{125}, 318$ (1957).
${ }^{4}$ G. W. F. Drake and A. Dalgarno, Astrophys. J. Letters $\underline{152}, \operatorname{L12} 1$ (1968).

${ }^{5}$ G. W. F. Drake, G. A. Victor, and A. Dalgarno, Phys. Rev. 180, 25 (1969).

${ }^{6}$ O. Bely and P. Faucher, Astron. Astrophys. 1, 37 
(1969).

${ }^{7}$ A. H. Gabriel and C. Jordan, Nature 221, 947 (1969).

${ }^{8}$ H. R. Griem, Astrophys. J. Letters 156, L103 (1969).

${ }^{9}$ A. I. Akhiezer and V. B. Berestetskii, Quantum Electrodynamics (Interscience, New York, 1965), pp. 347-353.

${ }^{10} \mathrm{~A}$. Dalgarno and G. W. F. Drake, Chem. Phys. Letters $\underline{3}, 349$ (1969).

${ }^{11}$ G. W. F. Drake and A. Dalgarno, Phys. Rev. A 1 , 1325 (1970).

${ }^{12} \mathrm{R}$. W. Schmieder and R. Marrus, Phys. Rev. Letters 25, 1245 (1970).

${ }^{13}$ M. Mizushima, Phys. Rev. 134 , A833 (1964).

${ }^{14} \mathrm{H}$. A. Bethe and E. E. Salpeter, Quantum Mechanics of One-and Two-Electron Atoms (Springer, Berlin, 1957), p. 69.

${ }^{15}$ R. Karplus and N. M. Kroll, Phys. Rev. $\underline{77}, 536$

(1950); J. Schwinger, ibid. 82, 664 (1951).

${ }^{16} \mathrm{~W}$. Perl and V. Hughes, Phys. Rev. 91, 842 (1953).

${ }^{17}$ L. L. Foldy and S. A. Wouthuysen, Phys. Rev. 78 , 29 (1950).

${ }^{18} \mathrm{~A}$. Messiah, Quantum Mechanics (Wiley, New York, 1962), Vol. II, pp. 944-946.

${ }^{19} \mathrm{~A}$ double-commutator relation with $H_{\mathrm{NR}}$ [cf. Eq. (34)] can be used to transform the term involving $\omega^{2}$ with the result that (Ref. 25) $\left\langle{ }^{1} S\left|Q_{10}\right| 2{ }^{3} S\right\rangle=\mu_{B}\left\langle 1{ }^{1} S\right|$ $-\left(1 / 3 m^{2} c^{2}\right)\left(p_{1}^{2}-p_{2}^{2}\right)+\left(e^{2} / 3 m c^{2}\right)\left[\left(r_{1}^{2}-r_{2}^{2}\right) / r_{12}^{3}\right]\left|2^{3} s\right\rangle$. The above form does not involve $\omega$ and can be obtained directly from the operator derived by Perl and Hughes (Ref. 16 and earlier references therein) for the magnetic dipole moment of the $2{ }^{3} \mathrm{~S}$ state of helium, even though their derivation applies only to a uniform static $(\omega=0)$ magnetic field. For computational purposes, Eq. (35) is more convenient since the $r_{12}$ coordinate does not appear.

${ }^{20}$ C. W. Scherr and R. E. Knight, Rev. Mod. Phys. 35,436 (1963).

${ }^{21} \mathrm{H}$. R. Griem has recently published an erratum giving the correct $Z^{10}$ dependence for the M1 decay rate [Astrophys. J. Letters 161, L155 (1970)].

${ }^{22}$ F. F. Freeman, A. H. Gabriel, B. B. Jones, and C. Jordan, Proc. Roy. Soc. (London) (to be published); A. H. Gabriel and C. Jordan, Phys. Letters 32A, 166 (1970).

${ }^{23} \mathrm{H}$. A. Bethe and E. E. Salpeter, Quantum Mechanics of One- and Two-Electron Atoms (Springer, Berlin, 1957), p. 107.

${ }^{24}$ A. R. Edmonds, Angular Momentum in Quantum Mechanics (Princeton U. P., Princeton, N. J., 1960), p. 118.

${ }^{25} \mathrm{C}$. Schwartz (private communication).

\title{
Measurements of Lowest $S$-State Lifetimes of Gallium, Indium, and Thalium ${ }^{\dagger}$
}

\author{
Michael Norton* and Alan Gallagher \\ Joint Institute for Laboratory Astrophysics, University of Colorado, Boulder, Colorado 80302 \\ (Received 3 September 1970)
}

The lifetimes of the gallium $5{ }^{2} S_{1 / 2}$ state, the indium $6{ }^{2} S_{1 / 2}$ state, and the thallium $7{ }^{2} S_{1 / 2}$ state were measured using the zero-field level-crossing (Hanle-effect) technique. The lifetimes obtained were $(6.8 \pm 0.3) \times 10^{-9},(7.0 \pm 0.3) \times 10^{-9}$, and $(7.45 \pm 0.2) \times 10^{-9} \mathrm{sec}$, respectively Anomalous contributions to the level-crossing signals, from the wavelength dependence of the exciting light intensity and self-absorption by the fluorescing atomic beam, were investigated in detail.

\section{INTRODUCTION}

A short time ago most measured or calculated atomic oscillator strengths were of uncertain reliability. Fortunately this situation is rapidly changing as a result of more numerous efforts and some different approaches. The increasing emphasis on direct lifetime measurements, rather than on oscillator strengths times vapor density, has freed the measurements from vapor-pressure and plasma-condition uncertainties. Increasing computer capabilities have allowed more sophisticated wave-function calculations. Almost as important, the available experimental and theoretical information is very efficiently utilized by Wiese and his collaborators, ${ }^{1}$ who make extensive studies of regularities and consistencies.
At the moment, accurate lifetime measurements for the upper state of the principal resonance lines of the group-I and -II elements have been made by two independent techniques, level crossing or magnetic resonance and phase shift. ${ }^{2-4}$ The agreements are almost all within the quoted experimental uncertainties, which are typically a few percent. A variety of relative oscillator-strength measurements, which are independent of vapor density, can be normalized to these resonance-line values to provide an impressive number of reliable oscillator strengths for these elements. ${ }^{1}$ The measurements reported here allow several of the group-III elements to arrive in this same category of reliability. For these group-III elements as well, many relative values can be tied down with a single good lifetime measurement, while the lifetimes measured 